\title{
Model of the Self-Q-Switching Instability of Passively Phased Fiber Laser Arrays
}

\author{
Erik J. Bochove, Alejandro B. Aceves, Yehuda Braiman, Pere Colet, Ralf Deiterding, Adrián Jacobo, \\ Casey A. Miller, Charley Rhodes and Sami A. Shakir
}

\begin{abstract}
We present a simple model for self-pulsation instability in passively phased high power optical fiber amplifier arrays with external feedback. Its key features are, first, the feedback level's sensitivity, and thus that of the cavity Q-value, to small phase changes of the array fields, and, second, the effect of refractive index nonlinearity in the amplifiers. The model's prediction of an instability threshold for arrays of at least two amplifiers is confirmed by a linearized stability analysis of a system in ring-cavity geometry, and the magnitudes of predicted power levels are well within the domain of recent experiments.
\end{abstract}

Index Terms - fiber lasers, passively phased fiber amplifier arrays, spatial filtering

\section{INTRODUCTION}

The goal of scaling fiber lasers to high power levels for industrial and military applications [1] has created an interest in coherently combining the beams of a number of fiber lasers or amplifiers [2]. However, success in accurately controlling the frequencies and phases by either active [3],[4] or passive techniques that include Talbot cavities [5], multicore fibers [5][8], Michelson cavities [9],[10],[11], fiber couplers [12],[13], Fourier cavities [14],[15], self-imaging resonators [16], fused fiber couplers [17], diffractive optics [18], and hybrid active-passive [19], among others, has until now been limited. Passive combining

Manuscript received October 9, 2010. This work was supported in part by the High Energy Laser Joint Technology Office (HELJTO) and the Air Force Office of Scientific Research (AFOSR).

Erik Bochove is with the Directed Energy Directorate, Air Force Research Laboratory, Kirtland Air Force Base, New Mexico 87117.

Alejandro Aceves is with the Department of Mathematics, Southern Methodist University, Dallas, Texas 75275.

Yehuda Braiman, Casey Miller and Ralf Deiterding are in the

Computer Science and Mathematics Division, Oak Ridge, National Laboratory, Oak Ridge, TN. Yehuda Braiman is also in the Department of Mechanical, Aerospace, and Biomedical Engineering of the University of Tennessee in Knoxville, Tennessee 37996, and

Casey Miller is a senior there in the Department of Mathematics.

Pere Colet and Adrián Jacobo are with IFISC (CSIC-UIB),

Campus Universitat de les Illes Balears, E07122 Palma de Mallorca, Spain.

Charley Rhodes is with Libration Systems Management, Inc., Albuquerque, New Mexico 87106.

Sami Shakir is with TASC, Inc., Albuquerque, New Mexico 87106. is nevertheless attractive because it does not require extensive optoelectronic feedback loops, so that it is a potentially simpler and robuster technology. However, passive methods are even at low power limited to small array sizes as consequence of inevitable optical path errors [20], [21], while at elevated powers pulsation instabilities arise [22]. A few analyses of the dynamics of passively phased fiber amplifier arrays have been published [23], [24] but the understanding of that is incomplete.

In this paper we extend the range of those studies to include relaxation oscillations, and that of our previous work [25] by including nonlinear phase effects. The latter will be shown to play an important role. Our focus is on a specific dynamic instability that is innate as well as unique to the passive phasing of laser arrays. We use a unified resonator approach to describe the dynamics of rare-earth-doped fiber amplifier arrays that are mutually locked in phase by coherent interaction in an external cavity. For concreteness we chose the ring-geometry spatiallyfiltering array system studied in [22], illustrated in Fig.1. We find that the set of differential equations describing this system has a stable fixed-point solution corresponding to a quiescent output field, until at elevated power levels an instability sets in that coincides with the onset of significant phase shifts due to index nonlinearity. A physical understanding of the mechanisms leading to this

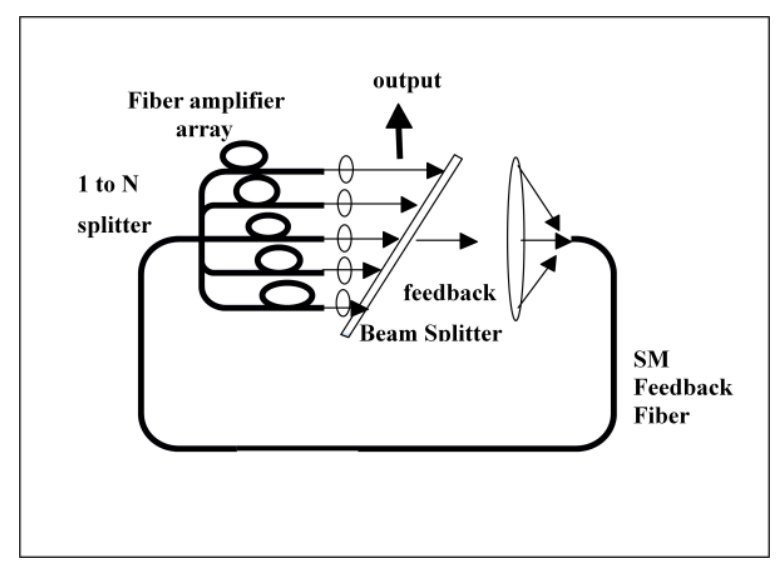

Fig.1. Diagram of passive phasing apparatus that serves as model for this paper [22],[25],[26]. 
instability is gained from the following argument that generally applies to any passive phasing systems in which phase-sensitive feedback is used as mechanism to control mode selection:

Suppose the device initially operates in a synchronous, quiescent, continuous wave state, which for this system is characterized by minimal cavity loss and optimum beam quality. Now assume the onset of a tiny initial perturbation that causes a momentary fluctuation in the phase of the output field of that amplifier. This would produce a proportionate loss in the strength of the feedback signal, since the ideal coupling condition, which relies on perfect array synchronization, is momentarily broken. A tiny intensity disturbance (consisting of a darkening of the bright back ground) then travels unhindered along the single-mode feedback fiber (SMF) and couples back into the amplifier array at its input plane, where it generates a small fluctuation in the refractive index due to the nonlinear properties of the medium. This in turn generates a secondary phase disturbance that if smaller than the original, will gradually decay on each roundtrip of the cavity, so that the system would then be stable. On the other hand, if the secondary disturbance exceeds the original, then it will selfregenerate on each successive round-trip, so that the system would in that case be dynamically unstable. The subsequent interruption of feedback can result in enough buildup of population inversion that it will be followed by emission of a sufficiently intense pulse that it could damage the system. Since these events involve a momentary increase of cavity loss (the induced decrease in feedback is associated with a loss increase), we refer to it as a "self Q-switching" effect.

The analysis developed next will lead to conclusions that support all aspects of the above argument and, moreover, predict transition regimes at power levels that appear to be consistent with experimental reports.

\section{MATHEMATICAL MODEL}

\section{A. Description of fields and gain media}

Let $E_{n}(z, t)=\mathcal{E}_{n}(z, t) \exp \left[i\left(\beta_{0} z-\omega_{0} t\right)\right]$ represent the on-axis laser field in each amplifier, where the index $n=1,2, . ., N$ labels the amplifiers, $\beta_{0}$ is the $L P_{01}$ mode propagation constant at central frequency $\omega_{0}$. The slowly-varying amplitudes $\mathcal{E}_{n}(z, t)$ satisfy a unidirectional nonlinear wave equation [25],

$$
\begin{aligned}
& \frac{\partial \mathcal{E}_{n}(z, t)}{\partial z}+\left(\beta_{0}{ }^{\prime}-\frac{1}{2} i g_{n}{ }^{\prime}(z, t)\right) \frac{\partial \mathcal{E}_{n}(z, t)}{\partial t}+ \\
& \left(\frac{1}{2} i \beta_{0}{ }^{\prime \prime}+\frac{1}{4} g_{n}{ }^{\prime \prime}(z, t)\right) \frac{\partial^{2} \mathcal{E}_{n}(z, t)}{\partial t^{2}}= \\
& \left(\frac{1}{2} g_{n}(z, t)+i k_{0} \Delta n_{N L ; n}(z, t)\right) \mathcal{E}_{n}(z, t)+\mathscr{R}_{n}(z, t),
\end{aligned}
$$

in which remaining symbols are defined as follows:

$$
\begin{aligned}
& \mathrm{g}_{n}(z, t)=\text { gain at central frequency } \omega_{0}, \\
& \Delta n_{N L ; n}(z, t)=\text { change in nonlinear refractive index, } \\
& \mathscr{R}_{n}(z, t)=\text { zero-mean stochastic function modeling } \\
& \quad \text { spontaneous emission, } \\
& k_{0}=\omega_{0} / c=\text { free-space propagation constant, } \\
& \quad \text { where } c=\text { speed of light, } \\
& \beta_{0}{ }^{\prime}, g_{n}{ }^{\prime} \text {, etc., denote derivatives of } \beta_{0}, g_{n} \text { with } \\
& \text { respect to frequency, where } \beta_{0}{ }^{\prime}=\text { inverse of group } \\
& \text { velocity, and } \beta_{0}{ }^{\prime \prime}=\text { group delay dispersion. }
\end{aligned}
$$

The units of $\mathcal{E}_{n}(z, t)$ are chosen such that $I_{s n}(z, t)=$ $\left|\mathcal{E}_{n}(z, t)\right|^{2}$ represents the signal intensity. Eq.(1) takes gain and group dispersion into account by analytical expansion of gain and propagation constant as functions of frequency about $\omega_{0}$, leading to the terms in the higher-order time derivatives in (1). The latter should be near the peak in gain, so that $g_{n}{ }^{\prime}(z, t) \approx 0$ and $g_{n}{ }^{\prime \prime}(z, t)<0$, these conditions being approximate because of the gain's dependence on the timedependent atomic level populations, discussed next.

The gain media in the ytterbium-doped fiber cores are modeled by two Stark-split level manifolds [27] described by lower and upper manifold populations $N_{l n}(\mathrm{z}, \mathrm{t})$ and $N_{2 n}(z, t)$, respectively, where their sum equals the $\mathrm{Yb}$-ion concentration, $N_{0}$. We assume that all processes of interest are slow compared to thermal relaxation rates, so that the sublevel populations of each manifold remain in thermal equilibrium with the surrounding medium.

The gain at frequency $\omega_{0}$ is given in terms of the populations by

$$
g_{n}(z, t)=\Gamma_{s}\left(\sigma_{e}\left(\omega_{0}\right) N_{2 n}(z, t)-\sigma_{a}\left(\omega_{0}\right) N_{1 n}(z, t)\right),
$$

where $\sigma_{e / a}\left(\omega_{0}\right)$ are effective emission and absorption cross-sections, and $\Gamma_{s}$ is the signal-core overlap factor. The population dynamics are represented by a rate equation model, 
$\frac{\partial N_{2 n}(z, t)}{\partial t}=R_{p ; n}(z, t)-g_{n}(z, t) \frac{I_{s n}(z, t)}{\varepsilon_{s} \Gamma_{s}}-\frac{N_{2 n}(z, t)}{\tau}$,

where $R_{p ; n}(z, t)$ is the pump-induced transition rate, $\varepsilon_{s}$ is the line-center photon energy, and $\tau$ is the upper-manifold life-time. The second term on the right-hand side of (3), with $g_{n}(z, t)$ given by (2), gives the transition rates due to absorption and stimulated emission of signal radiation. Last, we give $R_{p ; n}(z, t)$ in terms of the pump intensity, $I_{p ; n}(z, t)$ and effective cross-sections at pump frequency $\omega_{p}$ :

$R_{p ; n}(z, t)=\left(\sigma_{a}\left(\omega_{p}\right) N_{l n}(z, t)-\sigma_{e}\left(\omega_{p}\right) N_{2 n}(z, t)\right) I_{p ; n}(z, t) / \varepsilon_{p}$,

where $\varepsilon_{p}=\hbar \omega_{p}$ is the pump photon energy.

The nonlinear index increment in (1) consists of the sum of the Kerr and gain-related terms, the former given by (omitting subscripts $n$ ) $\Delta n_{\text {Kerr }}=n_{2} I_{s}(z, t)$ [28], and the latter represents the index change, related by Kramers-Kronig relations [29] to the imaginary part of the index, and in terms of the gain by $\operatorname{Im}(\Delta n)=c g(\omega) / 2 \omega$. Eq. (2) yields $\Delta n_{K K}(\omega)=J(\omega)$ $\times N_{2}(z, t)$, apart from a population-independent constant, where $J(\omega) \equiv \partial n(\omega) / \partial N_{2}$ is independent of ion concentration. For silicate we take $n_{2}=3.2 \cdot 10^{-20}$ $\mathrm{m}^{2} / W$ [28], and using tabulated data for the effective cross sections, we obtain $J \approx 3 \cdot 10^{-32} \mathrm{~m}^{3}$ at $1080 \mathrm{~nm}$, the laser wavelength used in our calculations, corresponding to a Henry parameter [30] $\alpha_{H}=-\operatorname{Re} \Delta n / \operatorname{Im} \Delta n$ close to unity.

The output fields of the array, $E_{m}\left(L_{m}, t\right)$, are focused into the core of the passive SMF [22], [26], where each amplifier contributes a term $U_{m} E_{m}\left(L_{m}, t-s_{m}\right)$ to the core field, where $U_{m}$ are coupling coefficients and $s_{m}$ time delays associated with the coupling optics. The SMF output is injected into the array input using a 1-N coupler, each leg described by coupling coefficient $V_{n}$ and delay $t_{n}$. This yields a linear relation between input and timedelayed output fields [25],

$$
E_{n}(0, t)=\sum_{m=1}^{N} V_{n} U_{m} E_{m}\left(L_{m}, t-\tau_{n m}\right),
$$

where $\tau_{n m}=t_{n}+s_{m}$. From (5) we obtain the sole cavity mode $\mathcal{F}$, described at array output by fields

$$
\mathscr{F}_{n}\left(L_{n}\right)=V_{n} e^{i \omega_{0} t_{n}} e^{i \beta_{0} L_{n}}
$$

This field corresponds to the eigenvalue

$$
\xi=\sum_{n=1}^{N} V_{n} U_{n} e^{i \omega_{0} \tau} c_{c n n},
$$

where $\tau_{c \mid n m}=\beta_{0} L_{m} / \omega_{0}+\tau_{n m}$. Eq.(7) shows that $|\xi|$ attains maxima at values of $\omega_{0}$ approximately satisfying

$$
\exp \left(i \omega_{0} \tau_{c \mid n n}\right) \sim 1 .
$$

This imposes a phasing condition upon the set $\mathcal{F}_{n}\left(L_{n}\right)$, provided $s_{1} \approx s_{2} \approx \ldots \approx s_{N}$, the physical reason of which is easily understood.

Figs.2a,b illustrate the passive phasing mechanism in terms of cold-cavity properties. Shown are plots of $|\xi|$ (solid curves) and an "order parameter" (dashed curves) that is a measure of phase quality, defined for an arbitrary set of fields $\mathcal{E}=\mathcal{E}_{1}, \mathcal{E}_{2}, \ldots, \mathcal{E}_{N}$ by

$$
\Omega(\mathcal{E})=\left|\sum_{n=1}^{N} \mathcal{E}_{n}\right| / \sum_{m=1}^{N}\left|\mathcal{E}_{n}\right|,
$$

which satisfies $\Omega(\mathcal{E}) \leq 1$. In the present application, $\mathcal{E}_{n}=\mathscr{F}_{n}\left(L_{n}\right)$. The scale of $|\xi|$ is arbitrary, since of main interest are the locations of the peaks, which coincide in Fig.2a where all $s_{n}$ are identical, and they do not coincide in Fig.2b, where that condition is violated. The laser system does not compensate for optical path errors in the section of the resonator between the output plane of the array and the input to the SMF, while it does so in part for path errors within the array and the 1-N coupler from the SMF to its input. It is thus important that those be reduced by minimizing the array dimensions and reducing offaxis aberrations of the focusing lens.

\section{B. Steady state solutions}

Assuming a steady-state solution of (1) \& (3) for which $\mathscr{R}_{n}(z, t)$ and $\partial / \partial t \rightarrow 0$, and separating (5) into real and imaginary parts, it yields the steady-state conditions

$$
\sum_{m=1}^{N} C_{n m}=1, \quad \sum_{m=1}^{N} S_{n m}=0,
$$

where 


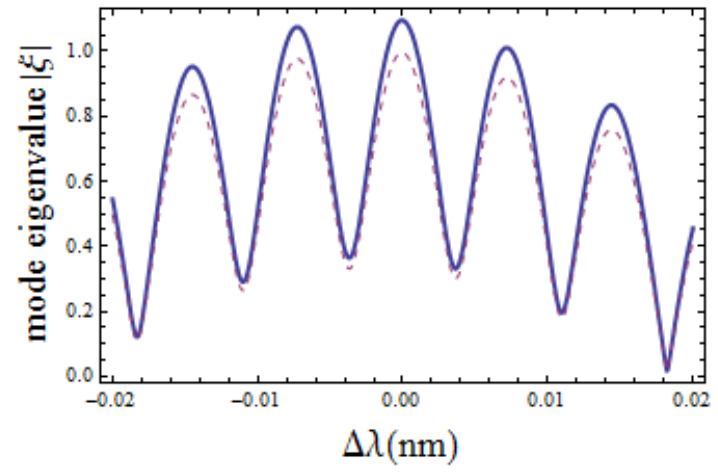

Fig.2a. Cavity eigenvalue (solid) with arbitrary normalization and order parameter $\Omega$ for $N=3$ system with fiber lengths $10,9.9$, and $10.013 \mathrm{~m}$ but with equal external path lengths $c s_{n}$.

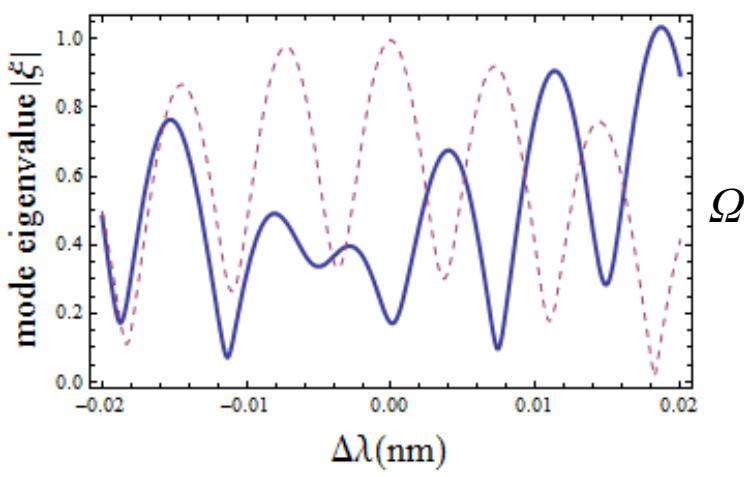

Fig.2b. Same system as represented in Fig.2a, but with external path length differences $c \Delta s_{n}=0,0.005,0.00233 \mathrm{~m}$

$$
\begin{gathered}
C_{n m}\left(S_{n m}\right)=V_{n} U_{m} \sqrt{I_{m}\left(L_{m}\right) / I_{n}(0)} \\
\times \cos (\sin )\left(\Phi_{m}\left(L_{m}\right)-\Phi_{n}(0)+\omega_{0} \tau_{c \mid n m}\right) .
\end{gathered}
$$

which will later be shown to describe the transport of fluctuations through the SMF between amplifiers $m$ and $n$. Using (1), the steady-state intensity $I_{n}(\mathrm{z})$ and phase $\Phi_{n}(z) \equiv \operatorname{Arg}\left[\mathcal{E}_{n}(z)\right]$ satisfy

$$
\begin{aligned}
& \frac{\partial I_{n}(z)}{\partial z}=g_{n}(z) I_{n}(z), \\
& \frac{\partial \Phi_{n}(z)}{\partial z}=k_{0} \Delta n_{N L ; n}(z) .
\end{aligned}
$$

Figs.2c,d show plots of the normalized total output power (solid lines), defined $P=\sum_{n=1}^{N}\left|\mathcal{E}_{n}\left(L_{n}\right)\right|^{2}$, and of the order parameter (dashed) of a cavity pumped well above threshold, with matched external delays, $s_{m}$,

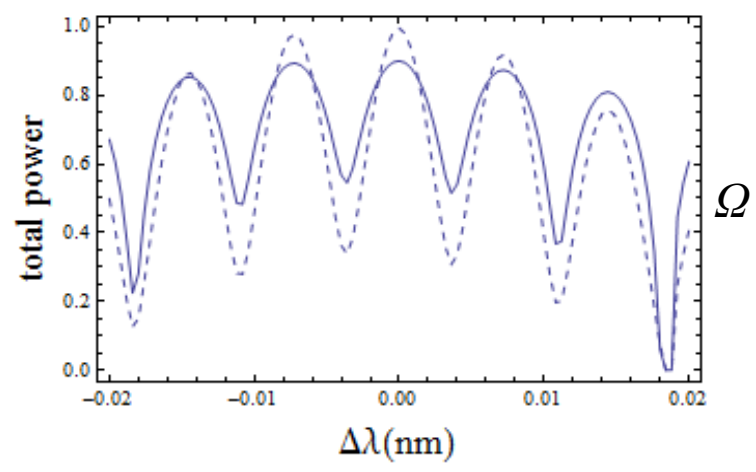

Fig.2c. Normalized total output power (solid) and order parameter $\Omega$ (dashed) of the system of Fig.2a when pumped well above threshold.

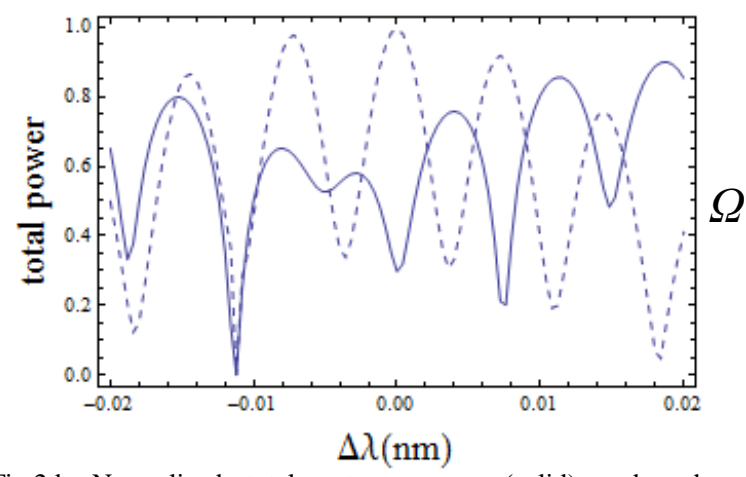

Fig.2d. Normalized total output power (solid) and order parameter $\Omega$ (dashed) of the system of Fig.2b when pumped well above threshold.

assumed in Fig.2c, and unmatched in Fig.2d. The solutions were obtained by numerical solution of (10) and (12a,b), yielding $I_{n}\left(L_{n}\right)$ and $\Phi_{n}\left(L_{n}\right)$ in terms of $I_{n}(0)$ and $\Phi_{n}(0) . \quad \Omega$ was determined using (9) with the computed output fields. Note that the peaks of $P$ and $\Omega$ plots coincide in Fig.(2c), but not in Fig.(2d). More to the point, Fig.2c resembles Fig.2a, and Fig.2d resembles Fig.2b.

Increase of array size $N$ results in sharpening of the spikes in these figures, which may be expected to lead to the spectral narrowing and spectral instability observed in [12].

\section{First-order perturbation treatment}

We introduce small fluctuations about steady-state values by $N_{2 n}(z, t)=N_{2 n}(z)+\eta_{2 n}(z, t), I_{n}(z, t)=I_{n}(z) \times$ $\left(1+i_{n}(z)\right)$, and $\Phi_{n}(z, t) \equiv \operatorname{Arg}\left(\mathcal{E}_{n}(z, t)\right)=\Phi_{n}(z)+\varphi_{n}(z, t)$ Pump fluctuations are neglected. Substitution in Eqs.(1)-(4), we find that to $1^{\text {st }}$ order the fluctuations satisfy the following partial differential equations in each amplifier [25], where $0 \leq z \leq L_{n}$ : 


$$
\begin{aligned}
& \frac{\partial \eta_{n}(z, t)}{\partial t}=-\frac{1}{\tau}\left(1+\frac{I_{n}(z)}{I_{s a t}(z)}\right) \eta_{n}(z, t)-\frac{g_{n}(z) I_{n}(z)}{\varepsilon_{s} \Gamma_{s}} i_{n}(z, t), \\
& \frac{\partial i_{n}(z, t)}{\partial z}+\beta_{0}{ }^{\prime} \frac{\partial i_{n}(z, t)}{\partial t}=\sigma_{s} \eta_{n}(z, t)-\frac{1}{4} g_{n}{ }^{\prime \prime}(z) \frac{\partial^{2} i_{n}(z, t)}{\partial t^{2}} \\
& -g_{n}{ }^{\prime}(z) \frac{\partial \varphi_{n}(z, t)}{\partial t}+\beta_{0}{ }^{\prime \prime} \frac{\partial^{2} \varphi_{n}(z, t)}{\partial t^{2}}, \\
& \frac{\partial \varphi_{n}(z, t)}{\partial z}+\beta_{0}{ }^{\prime} \frac{\partial \varphi_{n}(z, t)}{\partial t}=\frac{1}{4} g_{n}(z)^{\prime} \frac{\partial i_{n}(z, t)}{\partial t} \\
& -\frac{1}{4} \beta_{0} " \frac{\partial^{2} i_{n}(z, t)}{\partial t^{2}}+\frac{\omega_{0}}{c}\left(J \eta_{n}(z, t)+n_{2} I_{n}(z) i_{n}(z, t)\right) \\
& -\frac{1}{4} g_{n}{ }^{\prime}(z) \frac{\partial^{2} \varphi_{n}(z, t)}{\partial t^{2}},
\end{aligned}
$$

From the boundary condition (5), we also obtain first-order relations between fluctuations at the array output and those at the input:

$$
\begin{aligned}
& i_{n}(0, t)= \\
& \sum_{m=1}^{N}\left[C_{n m} i_{m}\left(L_{m}, t-\tau_{n m}\right)-2 S_{n m} \varphi_{m}\left(L_{m}, t-\tau_{n m}\right)\right], \\
& \varphi_{n}(0, t)= \\
& \sum_{m=1}^{N}\left[\frac{1}{2} S_{n m} i_{m}\left(L_{m}, t-\tau_{n m}\right)+C_{n m} \varphi_{m}\left(L_{m}, t-\tau_{n m}\right)\right],
\end{aligned}
$$

where $C_{n m}$ and $S_{n m}$ were introduced in (10). The $S_{n m}$ are of special significance since they determine the cross-coupling between amplitude and phase fluctuations in the feedback loop.

Assuming time-dependence of the form $\eta(z, t)=\eta(z) \exp (\chi t)$, etc., in which $\chi$ is a complex constant, allows elimination of $\eta(z)$ and reduces $(13 \mathrm{~b}, \mathrm{c})$ to ordinary differential equations:

$$
\begin{aligned}
\eta_{n}(z)= & -\frac{\tau g_{n}(z) I_{n}(z)}{\varepsilon_{s} \Gamma_{s} B_{n}(z, \chi)} i_{n}(z), \\
\frac{\partial i_{n}(z)}{\partial z}= & -\left(\frac{g_{n}(z)}{B_{n}(z, \chi)} \frac{I_{n}(z)}{I_{s a t}(z)}+\beta_{0}{ }^{\prime} \chi+\frac{1}{4} g_{n}{ }^{\prime \prime}(z) \chi^{2}\right) i_{n}(z) \\
& -\left(g_{n}{ }^{\prime}(z) \chi-\beta_{0}{ }^{\prime \prime} \chi^{2}\right) \varphi_{n}(z),
\end{aligned}
$$

$$
\begin{aligned}
& \frac{\partial \varphi_{n}(z)}{\partial z}=\left(\frac{\omega_{0}}{c} n_{2} I_{n}(z)-\frac{\omega_{0} J \tau}{c \varepsilon_{s}} \frac{g_{n}(z) I_{n}(z)}{B_{n}(z, \chi)}+\frac{1}{4} g_{n}{ }^{\prime}(z) \chi\right. \\
& \left.\left.-\frac{1}{4} \beta_{0}{ }^{\prime} \chi^{2}\right) i_{n}(z)-\left(\beta_{0}{ }^{\prime} \chi+\frac{1}{4} g_{n}{ }^{\prime \prime}(z) \chi^{2}\right) \varphi_{n}(z)\right)
\end{aligned}
$$

where

$$
B_{n}(z, \chi)=1+\chi \tau_{p}(z)+I_{n}(z) / I_{\text {sat }}(z)
$$

describes saturation effects, in which

$$
\begin{aligned}
& \tau_{p}(z)=\tau /\left(1+I_{p}(z) / I_{p \mid s a t}\right), \\
& I_{s a t}(z)=\varepsilon_{s} / \tau_{p}(z) \sigma_{s}, I_{p \mid s a t}=\varepsilon_{p} / \tau \sigma_{p}, \\
& \text { and } \sigma_{s / p}=\sigma_{a}\left(\omega_{0 / p}\right)+\sigma_{e}\left(\omega_{O / p}\right) .
\end{aligned}
$$

The boundary conditions (14) similarly reduce to

$$
\begin{aligned}
& i_{n}(0)=\sum_{m=1}^{N} e^{-\chi \tau_{n m}}\left[C_{n m} i_{m}\left(L_{m}\right)-2 S_{n m} \varphi_{m}\left(L_{m}\right)\right], \\
& \varphi_{n}(0)=\sum_{m=1}^{N} e^{-\chi \tau_{n m}}\left[\frac{1}{2} S_{n m} i_{m}\left(L_{m}\right)+C_{n m} \varphi_{m}\left(L_{m}\right)\right] .
\end{aligned}
$$

These show that we need the fluctuations at $z=L_{n}$, in terms of their values at $z=0$. To that end, the solution of $(15 b, c)$ is expressed in the form

$$
\begin{aligned}
& i_{n}(z)=e^{-\chi \beta_{0}{ }^{\prime} z}\left(G_{11}^{n}(z, \chi) i_{n}(0)+G_{12}^{n}(z, \chi) \varphi_{n}(0)\right), \\
& \varphi_{n}(z)=e^{-\chi \beta_{0}{ }^{\prime} z}\left(G_{21}^{n}(z, \chi) i_{n}(0)+G_{22}^{n}(z, \chi) \varphi_{n}(0)\right) .
\end{aligned}
$$

where we separated the relatively rapidly varying factor $\exp \left(-\chi \beta_{0}{ }^{\prime} z\right) \quad\left(\right.$ since $\operatorname{Im}(\chi) \beta_{0}{ }^{\prime} \sim q \beta_{0} / \omega_{0} \tau_{c}$, where $q$ equals zero or a multiple of $2 L / \Delta L, L$ is roughly a fiber length, and $\Delta L$ a typical length difference). Note that $G_{12}^{n}$ and $G_{2 l}^{n}$ describe the conversion of a phase disturbance to an amplitude disturbance and the reverse, respectively.

Taking $z=L_{n}$ in (19), and substituting $i_{n}\left(L_{n}\right)$ and $\varphi_{n}\left(L_{n}\right)$ obtained from (19) into (18), the latter is expressed in matrix form as

$$
\mathbf{Z}_{N}(\chi) \mathbf{X}(0)=0
$$

where the $2 N$-dimensional column vector $\mathbf{X}(0)$ spans the two $N$-dimensional sub-spaces of intensity and phase fluctuations:

$$
\mathbf{X}(0)=\left[\begin{array}{c}
\mathbf{i}(0) \\
\boldsymbol{\varphi}(0)
\end{array}\right],
$$


where $\mathbf{i}(0)=\left[\begin{array}{c}i_{1}(0) \\ i_{2}(0) \\ \vdots \\ i_{N}(0)\end{array}\right], \boldsymbol{\varphi}(0)=\left[\begin{array}{c}\varphi_{1}(0) \\ \varphi_{2}(0) \\ \vdots \\ \varphi_{N}(0)\end{array}\right] . Z_{N}(\chi)$ is a

$2 N \times 2 N$ square matrix that we write in the form

$$
\mathbf{Z}_{N}(\chi)=\left[\begin{array}{ll}
\mathbf{Z}^{11}(\chi) & \mathbf{Z}^{12}(\chi) \\
\mathbf{Z}^{21}(\chi) & \mathbf{Z}^{22}(\chi)
\end{array}\right]
$$

where $\mathbf{Z}^{i j}(\chi)$ represent four $N \times N$ square matrices having components given by

$$
\begin{aligned}
& Z_{n m}^{11}(\chi)= \\
& e^{-\chi \tau_{c \mid n m}}\left(C_{n m} G_{11}^{m}\left(L_{m}, \chi\right)-2 S_{n m} G_{21}^{m}\left(L_{m}, \chi\right)\right)-\delta_{n m}, \\
& Z_{n m}^{12}(\chi)=e^{-\chi \tau_{c \mid n m}}\left(C_{n m} G_{12}^{m}\left(L_{m}, \chi\right)-2 S_{n m} G_{22}^{m}\left(L_{m}, \chi\right)\right), \\
& Z_{n m}^{21}(\chi)=e^{-\chi \tau_{c \mid n m}}\left(\frac{1}{2} S_{n m} G_{11}^{m}\left(L_{m}, \chi\right)+C_{n m} G_{21}^{m}\left(L_{m}, \chi\right)\right), \\
& Z_{n m}^{22}(\chi)= \\
& e^{-\chi \tau_{c \mid n m}}\left(\frac{1}{2} S_{n m} G_{12}^{m}\left(L_{m}, \chi\right)+C_{n m} G_{22}^{m}\left(L_{m}, \chi\right)\right)-\delta_{n m} .
\end{aligned}
$$

The solutions of (20) will be referred to as relaxation modes, or just "modes" if confusion with normal resonator modes is improbable. The associated eigenvalues, $\chi_{q}$, are the roots of the Jacobian determinant:

$$
\operatorname{det} \mathbf{Z}_{N}(\chi)=0
$$

We can deduce some properties of the solutions of (20) without explicit calculation. Since $G_{12}^{m}\left(L_{m}, \chi\right)$ is of order $\beta_{0}$ " it is negligible except for very large $\chi$. Also, since under the conditions discussed at the end of section A, the coefficients $S_{n m}$ are likewise anticipated to be small, we have

$$
Z_{n m}^{12}(\chi) \approx 0 .
$$

$\mathrm{Z}_{N}(\chi)$ is then approximately of triangular form, so that (20)-(23) yield

$$
\begin{aligned}
& Z^{11}(\chi) i(0) \approx 0, \\
& Z^{21}(\chi) i(0)+Z^{22}(\chi) \varphi(0) \approx 0,
\end{aligned}
$$

The non-trivial solutions of (26a), which satisfy $i(0) \neq 0$, also satisfy

$$
\begin{aligned}
& \operatorname{det} Z^{11}(\chi) \approx 0, \\
& \varphi(0) \approx-Z^{22}(\chi)^{-1} Z^{21}(\chi) i(0) .
\end{aligned}
$$

These solutions constitute a group of relaxation modes that are of mixed intensity-phase character, as indicated by (27b). For convenience, we named this group "M" (for "mixed") relaxation modes.

The trivial solutions of (26) satisfy,

$$
\begin{aligned}
& i(0) \approx 0, \\
& Z(\chi) \varphi(0) \approx 0, \\
& \operatorname{det} Z(\chi) \approx 0 .
\end{aligned}
$$

These are essentially pure phase fluctuations, to which we refer as " $\Phi$ " relaxation modes. Numerical solutions of (20) have confirmed the existence of the two mode groups for small arrays, but how well this classification applies to larger arrays depends on how well the latter satisfy (25). This remains to be seen.

We proceed now with evaluation of the coefficients $G_{i j}^{n}$. We restrict ourselves to relatively low orders of relaxation modes so that terms in $\beta_{0}{ }^{\prime}, g_{n}{ }^{\prime}(z)$ etc., can be neglected. This does not affect the solutions we are interested in, which have instabilities in modes of relatively low order compared to the frequencies characterizing the omitted terms. This approximation permits explicit expressions to be obtained for all coefficients $G_{i j}^{n}$ if in addition we neglect pump depletion, so that $\partial I_{p} / \partial z=0$, then $I_{\text {sat }}(z) \rightarrow I_{\text {sat }}$ and $\tau_{p}(z) \rightarrow \tau_{p}$ are independent of $z$.

Subject to the given conditions, integration of (15b) and using (19), yields

$G_{11}^{n}(z, \chi)=\frac{B_{n}(0, \chi)}{B_{n}(z, \chi)}, G_{12}^{n}(z, \chi)=0$.

$G_{21}^{n}(z, \chi)$ is evaluated by integrating (15c), as follows:

$$
\begin{aligned}
G_{21}^{n}(z, \chi)= & \frac{\omega_{0} n_{2}}{c} \int_{0}^{z} I_{n}\left(z^{\prime}\right) G_{11}^{n}\left(z^{\prime}, \chi\right) d z^{\prime} \\
& -\frac{\omega_{0} J \tau}{c \varepsilon_{s}} \int_{0}^{z} \frac{g_{n}\left(z^{\prime}\right) I_{n}\left(z^{\prime}\right)}{B_{n}\left(z^{\prime}, \chi\right)} G_{11}^{n}\left(z^{\prime}, \chi\right) d z^{\prime} .
\end{aligned}
$$

Carrying out the integrations yields,

$$
G_{21}^{n}(z, \chi)=\frac{\omega_{0} n_{2}}{c} \frac{I_{s a t} B_{n}(0, \chi)}{g_{0}}\left(\tau_{p} \chi \log \left(\frac{B_{n}(0, \chi)}{B_{n}(z, \chi)}\right)+\right.
$$




$$
\left.\frac{I_{n}(z)-I_{n}(0)}{I_{s a t}}\right)-\frac{\omega_{0} J \tau}{c \varepsilon_{s}}\left(1-\frac{B_{n}(0, \chi)}{B_{n}(z, \chi)}\right)
$$

For $G_{22}^{n}(z, \chi)$ we trivially obtain from (15c) and (19),

$$
G_{22}^{n}(z, \chi)=1
$$

\section{Application to single-element array}

For a globally coupled array of $N$ perfectly identical amplifiers, so that $L_{n}, s_{n}, t_{n} \rightarrow L, s, t$ and $\tau_{c \mid n m} \rightarrow \tau_{c}$ $\equiv \beta_{0} L / \omega_{0}+s+t$, we find $C_{n m}=N^{-1}$ and $S_{n m}=0$.

Eqs. (23) then reduce to

$$
\left.\begin{array}{c}
Z_{n m}^{11}(\chi)=N^{-1} e^{-\chi \tau_{c}} G_{11}^{m}\left(L_{m}, \chi\right)-\delta_{n m}, \\
Z_{n m}^{12}(\chi)=N^{-1} e^{-\chi \tau_{c}} G_{12}^{m}\left(L_{m}, \chi\right)=0, \\
Z_{n m}^{21}(\chi)=N^{-1} e^{-\chi \tau_{c}} G_{21}^{m}\left(L_{m}, \chi\right), \\
Z_{n m}^{22}(\chi)=N^{-1} e^{-\chi \tau_{c}} G_{22}^{m}\left(L_{m}, \chi\right)-\delta_{n m} \\
=N^{-1} e^{-\chi \tau_{c}}-\delta_{n m},
\end{array}\right\}
$$

For $N=1, \mathbf{Z}_{1}(\chi)$ becomes

$$
\mathbf{Z}_{1}(\chi)=\left[\begin{array}{cc}
e^{-\chi \tau_{c}} G_{11}^{1}(L, \chi)-1 & 0 \\
e^{-\chi \tau_{c}} G_{21}^{1}(L, \chi) & e^{-\chi \tau_{c}}-1
\end{array}\right]
$$

Because this is rigorously of triangular form, the characteristic equation for each mode group is obtained by equating the diagonal elements to zero. Defining $R_{f} \equiv\left(U_{1} V_{1}\right)^{2}$, then from (5), $R_{f}=I(0) / I(L)$; the "M" relaxation modes satisfy,

$$
e^{\chi \tau_{c}}=\frac{1+\chi \tau_{p}+I(0) / I_{s a t}}{1+\chi \tau_{p}+I(0) /\left(I_{s a t} R_{f}\right)} .
$$

For " $\Phi$ " relaxation modes, we have

$$
e^{\chi \tau_{c}}=1 .
$$

Since $R_{f}<1$, Eqs.(34) have no solutions for $\chi$ that have a positive real part, so that the single-fiber ring laser is predicted to have stable stationary states, which is consistent with the physical model.

The solutions of (34b) are undamped cavity modes:

$$
\chi_{q}= \pm \frac{2 q \pi}{\tau_{c}} i, q=0,1,2, \ldots
$$

This expression makes sense, because a pure phase fluctuation does not affect the state of the medium (within our approximation) either in propagation, or for $N=1$, in the feedback coupling.

E. Application to $N>1$ arrays

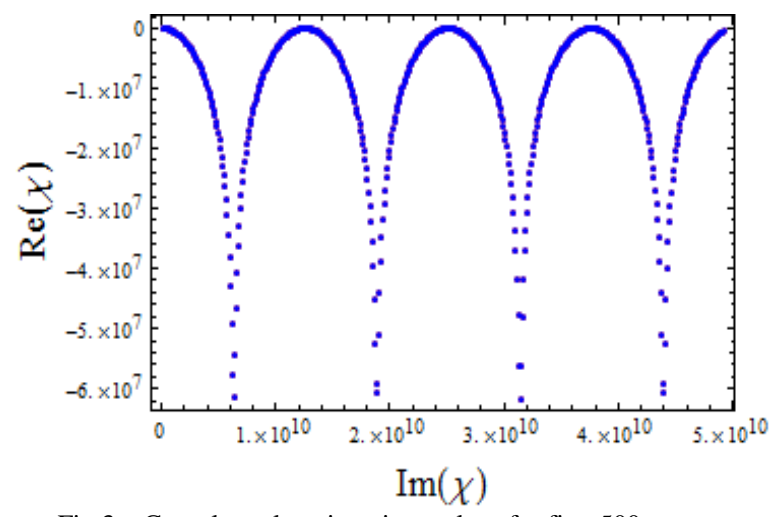

Fig.3. Complex relaxation eigenvalues for first 500 relaxation modes for a typical stable $N=2$ array

We now give numerical results for arrays of amplifiers of different lengths, although only the case of equal external delays, $s_{n}$, will be considered. These are sensitive to the location of $\omega_{0}$ relative to a peak in power, $P$. Unless $\omega_{0}$ is located at a peak, no stable stationary solutions were found, which may be explained by observing that for an off-peak value of $\omega_{0}$ the perturbation side-band nearest the peak is anticipated to have higher gain, so that the former serves as attractor. In the following figures, $\omega_{0}$ was always evaluated at a power peak.

Fig.3 shows a typical plot of the first 500 eigenvalues of an $N=2$ aray, obtained by numerical solution of (24). The case shown corresponds to a relatively low power level, making it stable, since all solutions satisfy $\operatorname{Re} \chi_{q}<0$. The fluted structure reflects the quasi-periodic nature of the resonator, for which the fiber lengths were arbitrarily chosen as $L_{1}=10 \mathrm{~m}, L_{2}=9.9 \mathrm{~m}$. In fact, the peaks in the figure correspond to peaks in the cavity eigenvalue as function of frequency. As the pump power is increased the peaks rise until solutions with positive real parts appear in increasing numbers. We use an $N=3$ array next to illustrate instability in detail.

Figs.4-6 for $N=3$ show clearly the effect of nonlinearities on stability, where the length of the additional amplifier is $10.013 \mathrm{~m}$. Fig. 4 was 


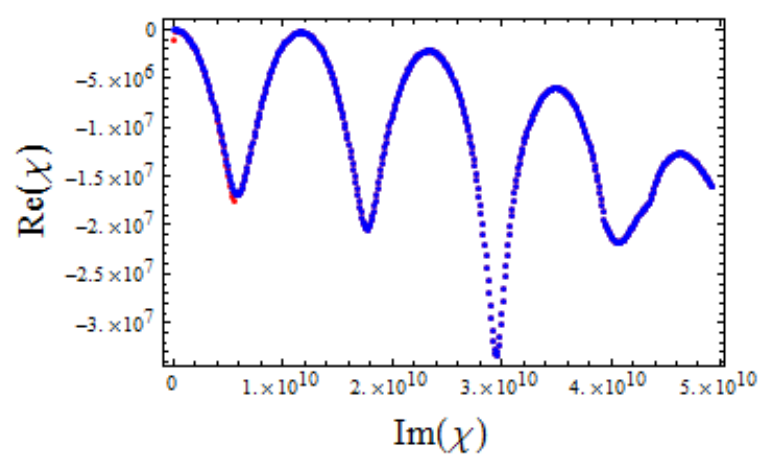

Fig.4. Complex eigenvalues of $N=3$ array, pumped at (unrealistic) $10 \mathrm{~kW}$ per fiber, for which the nonlinear constants were taken to vanish. All have negative real parts, implying dynamic stability.

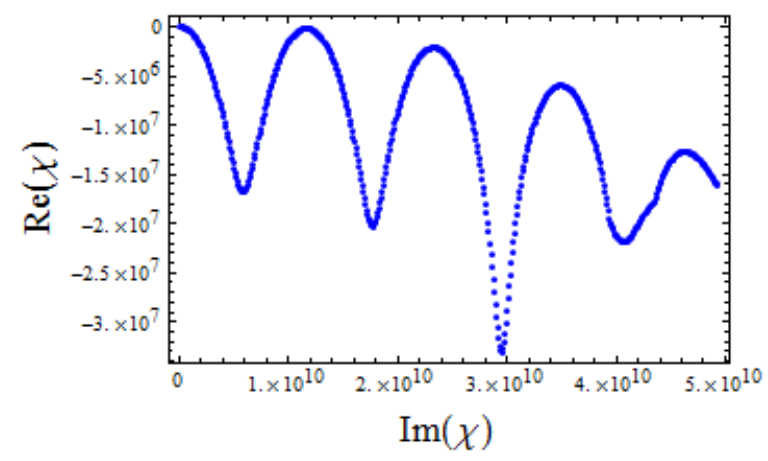

Fig.5. Complex eigenvalues of $N=3$ array with normal values of nonlinear constants, pumped at $50 \mathrm{~W}$ per fiber. The plot is essentially identical to Fig.4.

computed assuming purposefully unphysical values of the nonlinear constants and pump power, n.l. $n_{2}=J=0$ and $10 \mathrm{~kW}$ per amplifier, for reasons of illustration. Since all eigenvalues have negative real parts, the system is stable, even with the given high pump level (note that plots with lower pump levels were similar). Fig.5 shows the eigenvalues of the same three-element system, but with normal values for the nonlinear constants, and it is pumped at $50 \mathrm{~W}$ per amplifier. Since all eigenvalues have again negative real parts, it is again stable; in fact the two figures show no discernible difference.

The final figure, Fig.6, depicts the same system as Fig.5, also with actual values of the nonlinear constants, but it is pumped at $1 \mathrm{~kW}$ per amplifier. It is unambiguously seen to be unstable. The dynamics of the system would be chaotic in this case. Unstable relaxation modes actually began to appear at much lower powers (at a hundred or a few hundred watts in our calculations, which depends e.g. on array size), but if there are only a few such modes the interpretation is less clear, and properties like the variation between the peaks are not as evident. In

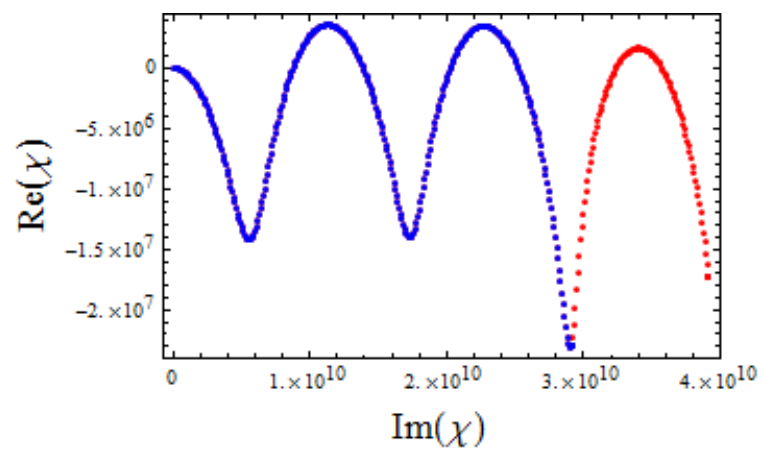

Fig.6. Complex eigenvalues of $N=3$ system pumped at $1 \mathrm{~kW}$, having normal values of nonlinear constants. Instability is indicated by the presence of eigenvalues with positive real parts.

particular, it was easier to distinguish effects that could be artifacts of numerical error from physically meaningful effects at high power. The results for the $N=1$ array and those shown in Figs.4-6 supply strong evidence in support of the physical model described in the Introduction. Moreover, it is significant that the predicted range of power levels at which the instabilities appear are in the neighborhood of experimental values, since if this theory were wrong these could easily be in disagreement by various orders of magnitude.

\section{DISCUSSION}

We proposed a physical mechanism for dynamic instability in passively phased high-power fiber amplifier arrays that is associated with nonlinear properties of the refractive index of the amplifier media. Since the mechanism involves collective array properties that relate the feedback efficiency by an external cavity to the phase synchronization of the array, this instability is intrinsic to arrays of at least two elements. We have referred to it as a self-Qswitching instability because, in contrast to conventional laser cavities [30], in this system the cavity Q-value is hyper-sensitive to fluctuations in the phases of the array fields.

The predictions of the physical model were confirmed by a first-order perturbation analysis of the continuous wave state of a ring-geometry cavity. This analysis, which includes interaction of the signal field and population fluctuations, but not the effect of pump fluctuations, predicted results in complete qualitative agreement with the physical model. In addition, the predicted power levels at which instability sets in appear to fall within the range of experimental observations. The physical model, however, strongly suggests that the described 
mechanism applies to a much wider range of passive phasing systems than the ring cavity modeled, including those of superposition architecture.

We have not presented the detailed properties of the relaxation modes, but one feature deserves mention. We noticed that the synchronous phasing quality tends to be maintained. This was observed experimentally [22], where in cases of chaotic output the reduction of side lobes in the far-field was maintained. In this case a predicted property close to steady state remains present when pulsations are more violent. Synchronization in the chaotic regime has been studied in arrays of semiconductor lasers [32].

Since we believe to have established in this paper a causal connection between nonlinearity and a predicted Q-switching instability, we suggest that mitigation of the latter can to a large measure be accomplished by known means of mitigating other nonlinear effects. These include the use of shorter fiber lengths, the reduction of the effective amplifier length by means of counter pumping [33], the use of large mode area (LMA) fibers [34], and multi-tone amplification [35], or other means. Some of those methods would also reduce the magnitude of the coupling elements, $S_{n m}$, such as by reduction of external path differences and nonlinearity of the amplifiers. Since all these suggestions have in common that they improve the quality of steady-state operation, by focusing on that requirement, one may also improve the prospects for dynamic stability.

Possessing full numerical solutions of the nonlinear equations is desirable, and we are working to develop the tools for that. However, we have not investigated the present linearized model to its full potential. The numerical solutions have been carried out for arrays of up-to only three amplifiers because of the significant numerical challenge involved, but treating larger arrays should be possible using other numerical techniques. At the single fixed fiberlength set, the three-element array was found to turn unstable at lower power levels than the two-element array, and we anticipate this to be a trend as the array size increases. Another limitation is that we have not reported on the comparative importance of the two kinds of index nonlinearity. Lastly, the investigation of the two relaxation mode groups that we labeled "M" and " $\Phi$ " is incomplete. The plots of Fig.3 onwards do not show that solutions came in pairs, but with separations that were too small to be resolved on the plots. This could be a manifestation of the above two mode groups, or an artifact of small numerical errors in the determination of the steady state solution. However, evidence that it may be the former is the presence of a slight kink in the plots of Figs.4 and 5. In other plots, two distinct branches would sometimes emerge from an apparent single branch, indicating the presence of a bifurcation, with one branch soon abruptly terminating. The latter suggests that the computer jumped between branches at that point due to insufficient precision in the choice of the initial estimate of the root, which could be resolved by refining our computational technique. We don't expect, however, that any of the conclusions reported here will be substantively altered.

\section{REFERENCES}

[1] D. J. Richardson, J. Nilsson, and W. A. Clarkson, "High power fiber lasers: current status and future perspectives," J. Opt. Soc. Am. B, Vol.27 (11), pp.B63-B90 (2010).

[2] T.Y. Fan, "Laser beam combining for high-power, highradiance sources," IEEE J. Sel. Topics Quantum Electron., Vol. 11, pp. 567-577 (2005).

[3] T. M. Shay, V. Benham, J. T. Baker, B. Ward, A. D. Sanchez, M. A. Culpepper, D. Pilkington, J. Spring, D. J. Nelson, and C. A. $\mathrm{Lu}$, "First experimental demonstration of self-synchronous phase locking of an optical array," Opt. Express, Vol.14, pp.1201512021 (2006).

[4] M. J. Wickham, P. Thielen, J. Ho, G. Goodno, R. Rice, E. Cheung and J. Rothenberg, "High efficiency coherent fiber beam combiner", Directed Energy Symposium, DEPS (2008).

[5] M. Wrage, P. Glas, D. Fischer, M. Leitner, D. V.Vysotsky, and A. P. Napartovich, "Phase locking in a multicore fiber laser by means of a Talbot resonator," Opt. Lett. 25, 1436-1438 (2000).

[6] P. K. Cheo, A. Liu, and G.G. King, "A high-brightness laser beam from a phase-locked multicore ytterbium-doped fiber laser array," IEEE Phot. Technol. Lett. Vol.13, pp. 439-441 (2001).

[7] L. Michaille, C. R. Bennett, D. M. Taylor, T. J. Shepherd, J. Broeng, H. R. Simonsen, and A. Petersson, "Phase locking, and supermode selection in multicore photonic crystal fiberlasers with a large doped area," Opt. Lett., Vol. 30, 1668-1670 (2005).

[8] L. Li, A. Schülzgen, S. Chen, V. L. Temyanko, J. V. Moloney, N. Peyghambarian,"Phase locking and in-phase supermode selection in monolithic multicore fiber lasers," Opt. Lett. , Vol. 31, 2577-2579 (2006).

[9] T. B. Simpson, A. Gavrielides, and P. Peterson, "Extraction characteristics of a dual fiber compound cavity," Opt. Express, Vol. 10, 1060-1073 (2002).

[10] M. Khajavikhan, K. John and J. Leger, "Experimental measurements of supermodes in superposition architectures for coherent laser beam combining", IEEE Journal of Quantum Electronics, Vol. 46, No. 8, 1221-1231, (2010).

[11] A. Ishaaya, N. Davidson and A. Friesem, "Passive laser beam combining with intracavity interferometric combiners", IEEE J. Sel. Top. Quant. Electron., Vol. 15, pp.301-311 (2009).

[12] A. Shirakawa, T. Saitou, T. Sekiguchi and K. Ueda, "Coherent addition of fiber lasers by use of a fiber coupler," Opt. Express, Vol. 10, pp. 1167-1172 (2002).

[13] A. Shirakawa, K. Matsuo, and K. Ueda, "Fiber laser coherent array for power scaling, bandwidth narrowing, and coherent beam direction control," Fiber Lasers II: Technology, Systems, and Applications, edited by L. N. Durvasula, A. J. W. Brown, J. Nilsson, Proc. SPIE Vol. 5709 (SPIE, Bellingham, WA, 2005)

[14] C. J. Corcoran and F. Durville, "Experimental demonstration of a phase-locked laser array using a self-Fourier cavity," Appl. Phys. Lett., Vol. 86, pp. 201118-1-201118-3 (2005).

[15] C. J. Corcoran and K. A. Pasch, "Modal analysis of a selfFourier laser cavity,” J. Opt. A: Pure Appl. Opt. 7, L1-L7 (2005). 
[16] Q. Peng, Y. Zhou, Y. Chen, Z. Sun, Y. Bo, X. Yang, Z. Xu, Y. Wang, K. Li, and W. Zhou, "Phase locking of fiber lasers by self imaging resonator," Electron. Lett, Vol. 41 (4) (2005).

[17] H. Bruesselbach, D. C. Jones, M. Mangir, M. Minden, and J. Rogers, "Self-organized coherence in fiber laser arrays," Opt. Lett., Vol. 301339 (2005).

[18] E. C. Cheung, J. G. Ho, G. D. Goodno, R. R. Rice, J. Rothenberg, P. Thielen, M. Weber, and M. Wickham, "Diffractiveoptics-based beam combination of a phase-locked fiber laser array," Opt. Lett., Vol. 33, pp. 354-356, (2008).

[19] X. L. Wang, P. Zhou, Y. X. Ma, H. T. Ma, X. J. Xu, Z. J. Liu, and Y. J. Zhao, "Coherent beam combining of pulsed fiber lasers with hybrid phase control", on-line available at http://www.springerlink.com/content/e876458m2r50q427/

[20] V. B. Gerasimov, M. V. Zakharov, V. V. Lyubimov, N. A. Makarov and V. K. Orlov, "Possibility of partial phase self-locking of retro-reflector elements in a resonator," Sov. J. Quantum Elect., Vol. 16, pp. 839-840 (1986).

[21] A. E. Siegman, "Resonant modes of linearly coupled multiple fiber laser structures," Unpublished manuscript (2004).

[22] S. T. Hendow, S. A. Shakir, W. Culver, and B. Nelson, "Passive phasing of fiber lasers," Solid State Diode Laser Technol. Rev. (SSDLTR), Albuquerque, NM (2007). See also, US Patent $7,130,113, \mathrm{~B} 2$.

[23]J. L. Rogers, S. Peleš, and K. Wiesenfeld, "Model for HighGain Fiber Laser Array", IEEE J. Quant. Electron., Vol. 41, pp. 767-773 (2005)

[24] T. Wu, W. Chang, A. Galvanauskas, and H. G. Winful, "Model for passive coherent beam combining in fiber laser arrays," Opt. Expr. Vol 17, pp. 19509-19518 (2009).

[25] E. Bochove, A. Aceves, R. Deiterding, L. Crabtree, Y. Braiman, A. Jacobo, P. Colet, "Space-time-dynamic model of passively-phased ring-geometry fiber laser array", Fiber Lasers VII: Technology, Systems, and Applications, edited by K. Tankala, J. W. Dawson, Proc. SPIE Vol. 7580 (2010).

[26] E. J. Bochove and S. A. Shakir, "Analysis of a SpatialFiltering Passive Fiber Laser Beam Combining System," IEEE J. Sel. Topics Quant. Electron. Vol.QE-15, pp. 320-327 (2009).

[27] R. Paschotta, J. Nilsson, A. C. Tropper, and D. C. Hanna,

"Ytterbium-Doped Fiber Amplifiers," IEEE J. of Quant. Electron., Vol. 33, pp.1049-1057 ( 1997).

[28] G. P. Agrawal, Nonlinear Fiber Optics, $4^{\text {th }}$ ed. New York: Academic (2007).

[29] L. D. Landau and E. M. Lifshitz, Electrodynamics of continuous media, Pergamon Press, Oxford, §62 (1960).

[30]C. H. Henry, "Theory of the Linewidth of Semiconductor Lasers," IEEE J. Quant. Electron. Vol. QE-18, pp.259-264 (1982).

[31] A. E. Siegman, Lasers, University Science Books, Sausalito (1986).

[ 32 ] H. G. Winful and L. Rahman, "Synchronized Chaos and Spatiotemporal Chaos in Arrays of Coupled Lasers," Phys. Rev. Lett. , Vol. 65, pp. 1575-1578 (1990)

[33] S. A. Shakir, Y. Starcher, E.J. Bochove, and C. Rhodes, "Suppression of stimulated Brillouin scattering and four-wavemixing in Yb-fiber amplifiers by counter-pumping." Manuscript in preparation (2010).

[34] J. C. Knight, T.A. Birks, R.F. Cregan, P.St. J. Russell and J.P. de Sandro" Large mode area photonic crystal fibre," Electron. Lett. Vol. 34, pp.1347-1348 (1998).

[35] C. Zeringue, I. Dajani, T. J. Bronder, T. Shay and C. Robin, "SBS mitigation with multi-tone amplification-a theoretical model," Proc. of SPIE, Vol. 7131, 71310Y-1.

Erik Bochove received the Ph.D. degree in physics from the University of Southern California, Los Angeles in 1974. During the 1970s he was a member of the Physics Department of the State University of Campinas (UNICAMP), Campinas, Brazil, where he helped develop fiber optical communications. He is currently a Senior Research Physicist at the Air Force Research Laboratory,
Albuquerque, New Mexico. His main current research interest is the modeling of passive phasing of high-power fiber laser arrays. $\mathrm{He}$ serves as Associate Editor for the IEEE Journal of Quantum Electronics.

Alejandro Aceves received his MSc in Applied Mathematics from the California Institute of Technology in 1983 and a $\mathrm{PhD}$ in Applied Mathematics from the University of Arizona in 1988. He is a Professor of Mathematics at Southern Methodist University. His research is on modeling nonlinear optical phenomena and has published over 50 publications in this area. In particular his research include the study of light localization in fiber arrays and the theoretical discovery of optical Bragg solitons.

Yehuda Braiman received his $\mathrm{PhD}$ degree from the University of Tel Aviv, Tel Aviv, Israel in 1993. He is a Distinguished Research Staff Member at the Center of Engineering Science Advanced Research (CESAR), Computer Science and Mathematics Division at Oak Ridge National Laboratory and a Joint Faculty Professor at the Department of Mechanical, Aerospace, and Biomedical Engineering at the University of Tennessee, Knoxville Tennessee. His most recent research topics include synchronization and coherent beam combining of high power arrays of semiconductor and fiber lasers. He also published papers in the fields of nonlinear dynamical systems and chaos control, friction, fracture, and dynamics of Josephson junctions. Dr. Braiman is a member of the American Physical Society and the Optical Society of America.

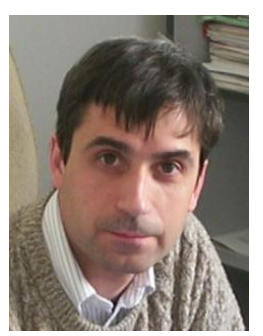

Pere Colet received his M.Sc. degree in physics in 1987 from the Universitat de Barcelona, and $\mathrm{Ph}$. D. degree in physics in 1991 from the Universitat de les Illes Balears, Palma de Mallorca, Spain. In 1991 he became teaching assistant at the Departament de Física of the Universitat de les Illes Balears. From September 1991 to February 1993 and from April to September 1994 he was a postdoctoral Fullbright fellow at the School of Physics of the Georgia Institute of Tecnology, Atlanta. In October 1994 he joined the Department de Física of the Universitat de les Illes Balears. Since May 1995 he has a permanent research position at the Spanish Consejo Superior de Investigaciones Cientificas. His present position is Research Professor at IFISC in Palma de Mallorca. He has coauthored over 100 papers in international journals as well as 30 other scientific publications. His research interests include fluctuations and nonlinear dynamics of semiconductor lasers, synchronization of chaotic lasers and encoded communications, synchronization of coupled nonlinear oscillators, pattern formation and quantum fluctuations in nonlinear optical cavities and dynamics of dissipative solitons.

Ralf Deiterding is a staff-level computational scientist and applied mathematician in the Computer Science and Mathematics Division at Oak Ridge National Laboratory (ORNL) . He is an expert in the areas of computational physics, numerical methods for partial differential equations, and high performance scientific computing. $\mathrm{He}$ obtained a PhD in 2003 from the Brandenburg Technical University Cottbus (Germany) and was a Postdoctoral Scholar in Applied and Computational Mathematics at the California Institute of Technology before joining ORNL in 2006.

Adrián Jacobo received the M.Sc. degree from the Universidad Nacional de Mar del Plata, Argentina, in 2003 and the Ph.D. degree in physics from the Universitat de les Illes Balears in 2009. Since October 2010, he holds a postdoctoral contract at the Max Plank Institute for the Physics of Complex Systems. His main research interests include nonlinear dynamics, spatiotemporal effects, and dynamics of localized structures. 
Casey Miller is a senior at the Department of Mathematics at the University of Tennessee, Knoxville. She is interested in the field of laser dynamics and synchronization. She is involved in fiber laser research since July 2009 as an intern at the Center for Engineering Science Advanced Research, Computer Science and Mathematics Division, Oak Ridge National Laboratory.

Charley Rhodes (M '06) received the Ph.D. in physics from New Mexico Tech in 1989. He was a Technical Staff Member at Los Alamos National Laboratory from 1990 to 2005 working on he USNDS system for detecting Nuclear EMP. He is currently Senior Scientist at Libration Systems Management in Albuquerque, NM working on the design of antennas and RF systems and supporting AFRL research on nonlinear effects in high-power fiber laser arrays

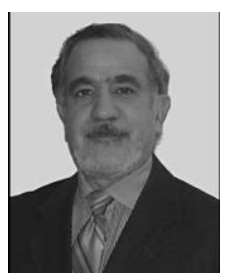

Sami Shakir received the B.S. degree in physics from Baghdad University, Baghdad, Iraq, in 1975, and the M.S. and Ph.D. degrees in optical sciences from the University of Arizona, Tucson, in 1978 and 1980, respectively. He is currently a Senior Scientist with TASC Inc. He was a Technical Fellow with Northrop Grumman until 2009. His current research interests include high power laser systems and fiber lasers, high-power laser beam propagation, and laser effects. 